\title{
High-voltage 10 ns delayed paired or bipolar pulses for in vitro bioelectric
} experiments

Orlacchio, Rosa ; Carr, Lynn; Palego, Cristiano; Arnaud-Cormos, Delia; Leveque, Philippe

\section{Bioelectrochemistry}

DOI:

10.1016/j.bioelechem.2020.107648

Published: 01/02/2021

Peer reviewed version

Cyswllt i'r cyhoeddiad / Link to publication

Dyfyniad o'r fersiwn a gyhoeddwyd / Citation for published version (APA):

Orlacchio, R., Carr, L., Palego, C., Arnaud-Cormos, D., \& Leveque, P. (2021). High-voltage 10 ns delayed paired or bipolar pulses for in vitro bioelectric experiments. Bioelectrochemistry, 137, [107648]. https://doi.org/10.1016/j.bioelechem.2020.107648

\footnotetext{
Hawliau Cyffredinol / General rights

Copyright and moral rights for the publications made accessible in the public portal are retained by the authors and/or other copyright owners and it is a condition of accessing publications that users recognise and abide by the legal requirements associated with these rights.

- Users may download and print one copy of any publication from the public portal for the purpose of private study or research.

- You may not further distribute the material or use it for any profit-making activity or commercial gain

- You may freely distribute the URL identifying the publication in the public portal ?
}

Take down policy

If you believe that this document breaches copyright please contact us providing details, and we will remove access to the work immediately and investigate your claim. 


\section{Journal Pre-proofs}

High-Voltage 10 ns Delayed Paired or Bipolar Pulses for In Vitro Bioelectric Experiments

Rosa Orlacchio, Lynn Carr, Cristiano Palego, Delia Arnaud-Cormos, Philippe Leveque

PII: S1567-5394(20)30206-1

DOI: https://doi.org/10.1016/j.bioelechem.2020.107648

Reference: BIOJEC 107648

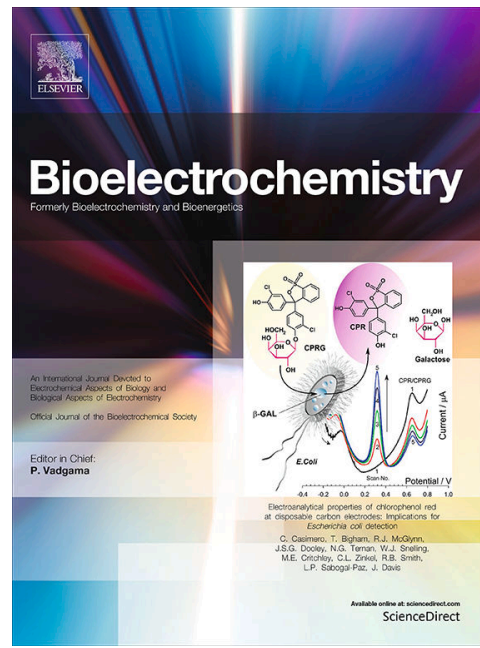

To appear in:

Bioelectrochemistry

Received Date:

6 April 2020

Revised Date:

31 August 2020

Accepted Date:

31 August 2020

Please cite this article as: R. Orlacchio, L. Carr, C. Palego, D. Arnaud-Cormos, P. Leveque, High-Voltage 10 ns Delayed Paired or Bipolar Pulses for In Vitro Bioelectric Experiments, Bioelectrochemistry (2020), doi: https:// doi.org/10.1016/j.bioelechem.2020.107648

This is a PDF file of an article that has undergone enhancements after acceptance, such as the addition of a cover page and metadata, and formatting for readability, but it is not yet the definitive version of record. This version will undergo additional copyediting, typesetting and review before it is published in its final form, but we are providing this version to give early visibility of the article. Please note that, during the production process, errors may be discovered which could affect the content, and all legal disclaimers that apply to the journal pertain.

(C) 2020 Published by Elsevier B.V. 


\title{
High-Voltage 10 ns Delayed Paired or Bipolar Pulses for In Vitro Bioelectric Experiments
}

\author{
Rosa Orlacchio ${ }^{1}$, Lynn Carr ${ }^{1,2}$, Cristiano Palego ${ }^{2}$, Delia Arnaud-Cormos ${ }^{1,3^{*}}$, Philippe \\ Leveque $^{1}$ \\ ${ }^{1}$ Univ. Limoges, CNRS, XLIM, UMR 7252, F-87000 Limoges, France \\ ${ }^{2}$ School of Electronic Engineering, Bangor University, Bangor, UK \\ ${ }^{3}$ Institut Universitaire de France (IUF), 75005, Paris France \\ *Corresponding author: Delia Arnaud-Cormos \\ Email : delia.arnaud-cormos@unilim.fr
}

\begin{abstract}
Recent studies proved that classical bio-effects induced by nanosecond pulsed electric field (nsPEF) can be reduced by the delivery of a negative polarity pulse generated immediately after a positive polarity pulse. This phenomenon is known as "bipolar cancellation" and it was reported for a wide range of bipolar pulses with pulse duration from $2 \mathrm{~ns}$ to $900 \mathrm{~ns}$. On the contrary, paired pulses, i.e., two identical pulses with the same polarity, increased traditional nsPEF outcomes. Herein, we propose a novel robust and flexible generator, based on the frozen-wave concept, able to produce a broad range of pulses with the duration of $10 \mathrm{~ns}$ and delay between 17 and $360 \mathrm{~ns}$. Numerical simulations and experimental measurements were performed to fully characterize the proposed generator. YO-PRO ${ }^{\mathrm{TM}}-1$ uptake was investigated in the U87-MG human glioblastoma cell line as a marker of membrane permeabilization in response to $10 \mathrm{~ns}, 11.5 \mathrm{MV} / \mathrm{m}$ nsPEF. Our results showed that bipolar cancellation occurred for delays of 0-30 ns and decreased as a function of the interphase interval. In addition, we observed that cellular response following the application of paired nsPEF was more than two-fold compared to the unipolar pulse response and was independent from the interphase interval.
\end{abstract}

\section{Keywords}

pulsed electric field; nsPEF; bipolar cancellation; versatile nanosecond generator; interphase pulse interval; YO-PRO ${ }^{\mathrm{TM}}-1$ uptake 


\section{Introduction}

In recent years, many studies have investigated the effects of bipolar pulses in the context of electroporation for biomedical applications. It was shown that bipolar pulses in the nanosecond range produce cancellation of the bioeffects classically observed when applying unipolar pulses [1]-[6]. This phenomenon was largely assessed with pulse durations from $60 \mathrm{~ns} \mathrm{[1],} \mathrm{[3],} \mathrm{[7]} \mathrm{to} 900 \mathrm{~ns}$ [8], and more recently for $2 \mathrm{~ns}$ [9], for different cell lines (CHO, Jurkat, U937, or excitable cells like chromaffin cells) and for a wide range of endpoints such as YO-PROTM-1 (YP) uptake, $\mathrm{Ca}^{2+}$ transients, cell death, calcein efflux, nerve excitation, or phosphatidylserine externalization. Cancellation with nanosecond pulsed electric fields (nsPEF) was observed with both symmetric, where the front pulse is followed by a negative polarity pulse of the same duration and amplitude [1], [4], [10] and asymmetric, where the front pulse is followed by a negative polarity pulse of different duration and/or amplitude [10]. For example, the delivery of a symmetric negative polarity pulse within a short interval of approximately $0.1-50 \mu \mathrm{s}$ after the positive polarity resulted in a reduced efficiency of electroporation compared to a single unipolar pulse [1], [4]. Similarly, traditional effects of nsPEF were cancelled by the exposure to asymmetric bipolar nsPEF when the amplitude and the duration of the second pulse were reduced to $35 \%$ [2] and 33\% [10] of the front pulse, respectively. Recently, Pakhomov et al [11] evaluated the efficiency of electroporation as a function of the second phase of the bipolar nsPEF with durations between $200 \mathrm{~ns}$ and $830 \mathrm{~ns}$. It was evident that maximum cancellation, or in other words the least electroporation, occurred when the amplitude of the pulse's second polarity was $50 \%$ of the first polarity. On the contrary, the application of a higher second phase resulted in the reduction or elimination of cancellation. Similarly, it was reported that a negative pulse three times the duration of the first caused complete loss of bipolar cancellation [10]. Moreover, the study revealed that cancellation is progressively reduced by increasing the interphase interval within the pulse [1], [4], and it can be completely reversed with a delay larger than $10 \mathrm{~ms}$ [8]. In addition, the delivery of two identical pulses of the same polarity (paired pulses) separated by a certain time gap was investigated. Additive or supra-additive effects were obtained on $\mathrm{YP}$ or $\mathrm{Tl}^{+}$ions uptakes or $\mathrm{Ca}^{2+}$ transients after exposure to single $600 \mathrm{~ns}$ or $300 \mathrm{~ns}$ paired pulses compared to a single $300 \mathrm{~ns}$, with different interpulse interval from 0.4 to $1000 \mu$ s [12]. Likewise, two paired pulses with single duration of 300 ns separated by $50 \mu$ s caused two-fold greater membrane permeabilization than a comparable bipolar nsPEF treatment [4].

Results of these studies strongly suggest that duration, amplitude, and delay of the reversed second pulse play an important role in determining the efficacy of cancellation. However, despite several formulated hypothesis [11], the mechanism responsible for cancellation is still under investigation. In addition, bipolar cancellation is characteristic of the nanosecond range, as it was not observed in conventional electroporation. Indeed, when the duration of the pulse is larger than $100 \mu \mathrm{s}$, the delivery of pulses of opposite polarity, known as high-frequency irreversible electroporation (H-FIRE), enhanced typical bio-effects [13]-[16] suggesting that a completely different mechanism is involved depending on the pulse duration. Therefore, the quantification of bipolar cancellation as a function of the pulse parameters may represent the key to unravel the mechanism underlying the occurrence of bipolar cancellation. Interphase intervals below $0.1 \mu$ s and pulse duration between 2 ns and $60 \mathrm{~ns}$ have not yet been fully explored.

These studies require the implementation of dedicated nsPEF generators able to allow flexibility in terms of pulse duration and amplitude as well as of the delay between two polarities (paired positive pulses or positive and negative polarities of bipolar pulses [17], [18]). Typical nsPEF generation techniques include Marx [19]-[21], magnetic pulse compression [22], [23], and Blumlein pulsed forming networks-based generators [24]-[26]. They are based on capacitive charging and fast switching using power components such as spark gap, power semiconductors such as metaloxide-semiconductor field-effect transistors (MOSFETs) [27], [28] or photoconductive semiconductor switches (PCSS) such as high voltage diode rectifiers used as optoelectronic switches [17], [29], [30].

We propose here a high-voltage nsPEF generator able to generate simultaneously two pulses of approximately $10 \mathrm{~ns}$ duration in this implementation with different time gaps. It is based on the frozen-wave principle using an optoelectronic technology. Depending on the generator setup, two paired pulses with positive polarity or bipolar pulses with the delayed negative polarity can be generated. To test the performance of the generator in operational conditions, in vitro experiments were carried out to assess the permeabilization of the U87-MG human glioblastoma cell line through YP uptake following the exposure to unipolar, bipolar, and paired nsPEF with no delay and interphase intervals up to $150 \mathrm{~ns}$. U87-MG is a widely used glioma cell line and glioblastoma multiforme is one of the major research interests of our laboratory [31]-[33]. As U87-MG are adherent, they are more appropriate for our electrode exposure system and imaging set up.

\section{Materials and Methods 2.1 Generation design and implementation}




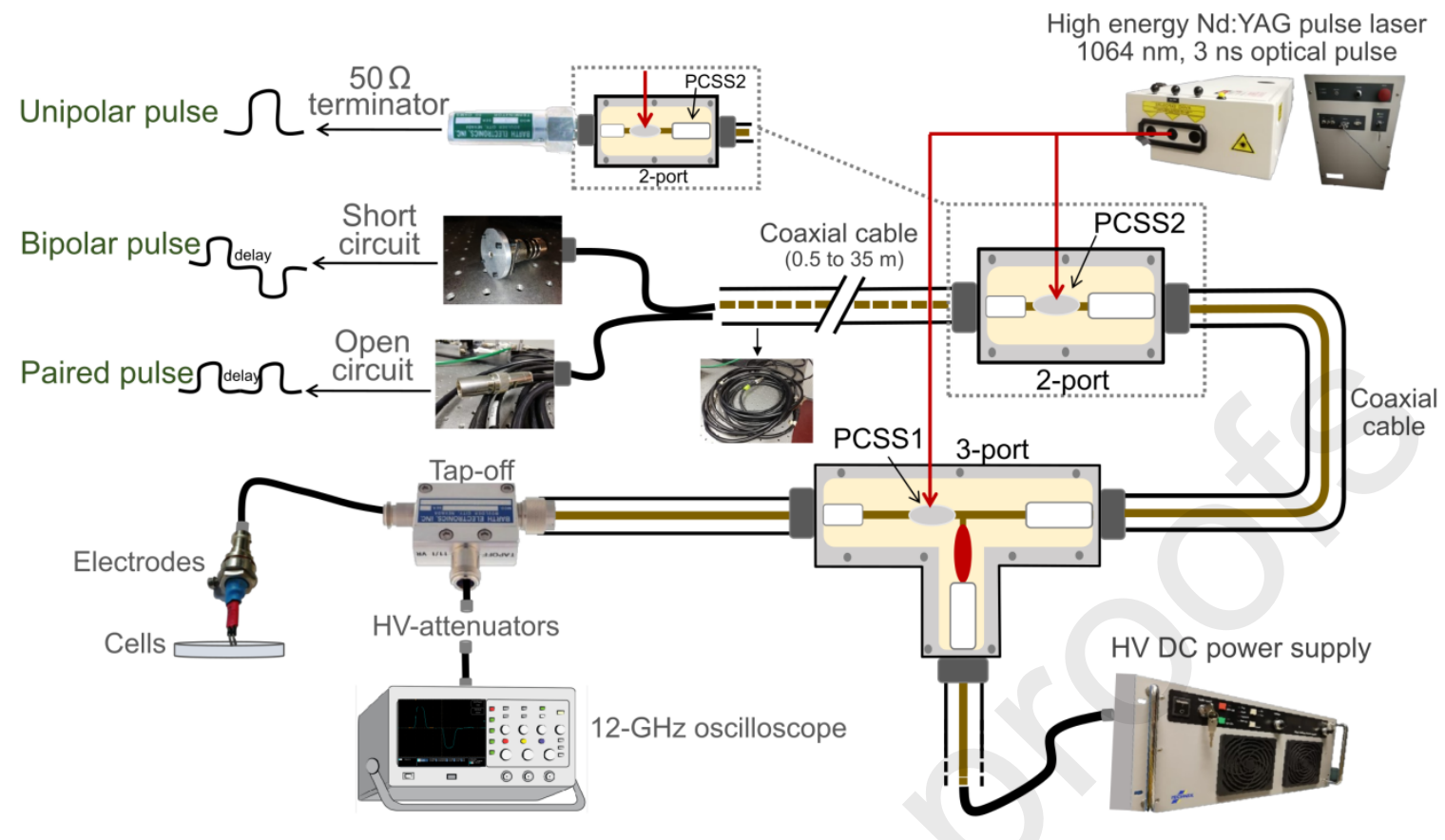

Fig. 1. Experimental setup for the generation and measurement of unipolar, bipolar, and paired high-voltage pulses. A high-voltage DC power supply releases energy into a transmission line (coaxial cables) acting as a capacitive storage component. Two photoconductive semiconductor switches (PCSSs) are integrated in the transmission lines within the 2- and 3-port boxes connected through a $2 \mathrm{~m}$ length coaxial cable for the generation of two $10 \mathrm{~ns}$ pulses. The delay between the pulses is established by the length of the cable at the output of port- 2 (in the $0.5-35 \mathrm{~m}$ range). A pulsed laser optically triggers the PCSSs, allowing the delivery of the stored energy into the biological cells through a pair of electrodes. The tap-off allows real-time measurement of the generated pulses visualized through a $12 \mathrm{GHz}$ oscilloscope (dimensions are not to scale).

Fig. 1 shows the implemented generator with unipolar and delayed negative or positive polarity nsPEF. This generator is based on the frozen-wave concept we used in previous setups and described in [34], [35]. The novelty of this generator consists in using a new designed 2-port coaxial box which introduces, through a transmission line of variable length, a time delay between the positive and the negative polarity of bipolar pulses, or between two positive polarities of paired pulses. In this implementation, two photoconductive semiconductor switches (PCSSs) are embedded in the 2- and 3- port coaxial boxes (PCSS2 and PCSS1 in Fig. 1), respectively. The PCSSs are high-voltage rectifier diodes sustaining up to $20 \mathrm{kV}$ DC (X200FG, VMI, USA). The PCSSs are triggered with a high-energy modelocked Nd:YAG laser (PL2241A, Ekspla, Lithuania) operating at $1064 \mathrm{~nm}$ and generating $2.4 \mathrm{~ns}$ optical pulses. The laser repetition rate was $20 \mathrm{~Hz}$ and its energy could reach $30 \mathrm{~mJ}$ per optical pulse that is sufficient for proper operation of the PCSSs. To operate both PCSSs, the laser beam is split in half and the simultaneous triggering is achieved using optical lines introducing equal delay. PCSSs are used in their linear mode thus avoiding temporal switching jitter for nanosecond duration pulses. PCSSs were characterized with time domain measurements. They typically transform from a high resistor of $33 \mathrm{M} \Omega$ while in the open state to a resistor of a few $\Omega$ when illuminated by a laser beam [29]. A high-voltage DC power supply (SR20kV-300W, Technix, France) was used to charge an electrical pulse on the 3port which is then released through the 2-port into a transmission line. The latter is terminated by a $50 \Omega$ terminator, short-circuit, or open-circuit to obtain unipolar, bipolar, or paired pulses, respectively. A high-voltage $50 \Omega$ terminator (Barth Electronics model 2051) was used to generate one positive polarity pulse. The short-circuit was made of a HN flange receptacle connector modified with a flat round metallic piece. On the short-circuit termination, a voltage pulse is reflected with an opposite phase producing a bipolar pulse. The open-circuit was created by ending the coaxial cable with a connector where the internal metallic wire was removed. On the open-circuit termination, a voltage pulse is reflected with the same phase producing a paired pulse.

The transmission line length at the output of the 2-port coaxial box determines the duration of the delay between the two pulses, while the length of the coaxial cable between the 2-and 3-port boxes establishes the duration of the pulses. In this study, to generate two $10 \mathrm{~ns}$-duration pulses, a $2 \mathrm{~m}$ cable length was used to connect the two ports, while up to $35 \mathrm{~m}$ length cable was used to obtain pulse delays up to $360 \mathrm{~ns}$. The 3-port coaxial box was connected using THT connectors (THT20, Radiall, France) sustaining up to $20 \mathrm{kV}$ to the power supply and to the 2-port coaxial box. To connect the 2-port and 3-port boxes to coaxial cables toward output, HN connectors (R176404, R176021, Radiall, 
France) were used. The boxes were designed to match the $50 \Omega$ characteristic impedance of THT, HN connectors, and RG214 coaxial cables. To avoid electrical discharges, the box was filled with vegetable oil.

The generated pulses were displayed on a $12 \mathrm{GHz}$ oscilloscope (DSO, TDS6124C, Tektronix, USA) through a tap-off (Model 245-NMFFP-100, Barth Electronics, Inc, NV, USA) connected between the generator and the electrodes using two transmission lines (RG214 coaxial cables). The tap-off is a three-port device whose mainline and measurement port impedances are $50 \Omega$ and $4950 \Omega$, respectively. This allows the voltage measurement on the oscilloscope with a 1:100 ratio, corresponding to $40 \mathrm{~dB}$ attenuation. Only $1 \%$ of the signal is extracted from the pulse propagating though the tap-off. Therefore, with negligible insertion losses, $99 \%$ of the pulse is transmitted to the output. In addition, $30 \mathrm{~dB}$ attenuation was added on the measurement port to provide the signal on the oscilloscope. Experimental high-voltage measurements of the forward and reflected pulses under various conditions were also made with the set-up shown Fig. 1.
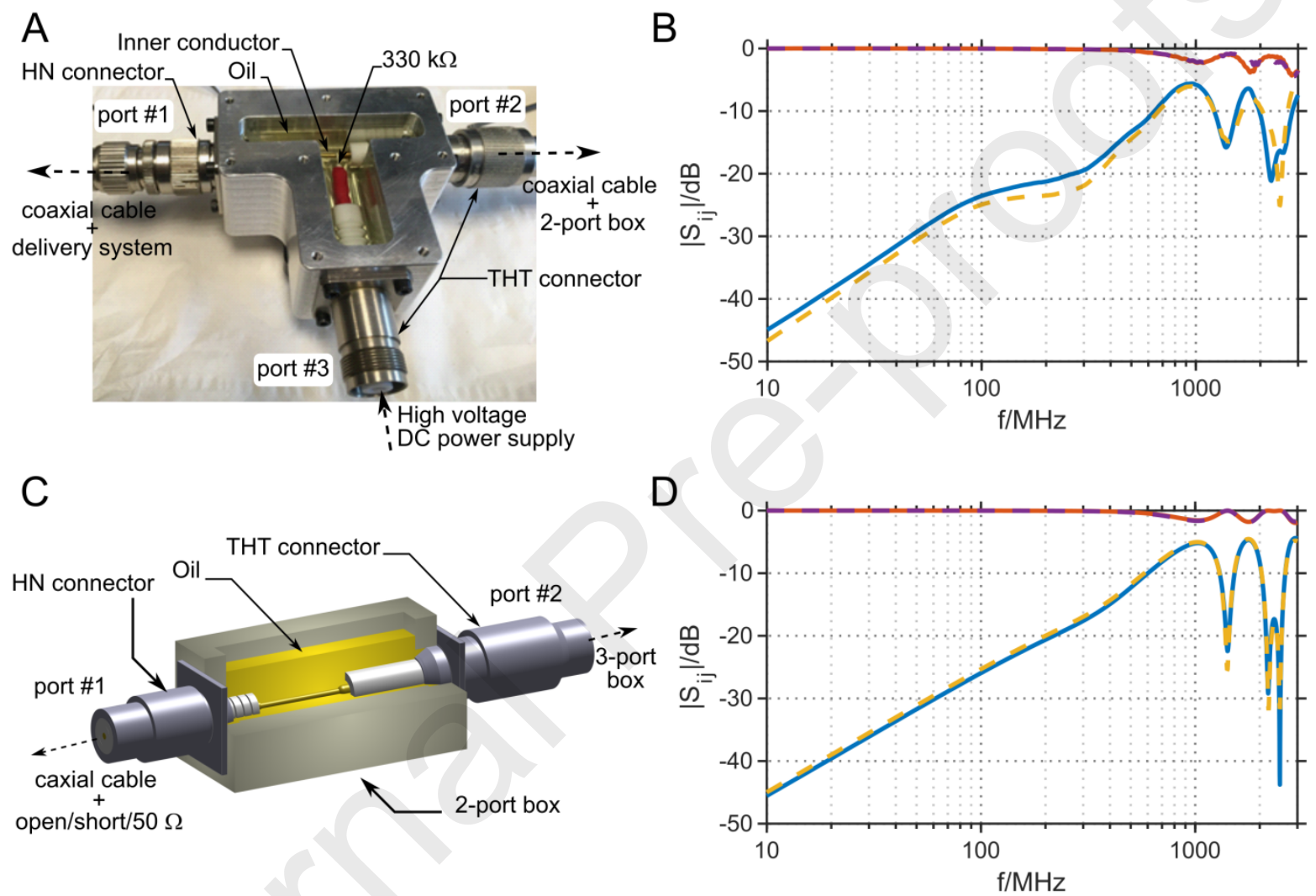

Fig. 2. Electromagnetic frequency characterization of the 2-port and 3-port coaxial boxes. A) Picture of the 3-port box filled with oil; port \#1 is the output port, port \#2 is connected to the 2-port box, and port \#3 is connected to the high voltage source though a $330 \mathrm{k} \Omega$ resistor (under the red rubber), B) Measured reflection $\left(\mathrm{S}_{11}\right)$ and transmission $\left(\mathrm{S}_{21}\right)$ parameters $\left(\left|\mathrm{S}_{\mathrm{ij}}\right| / \mathrm{dB}\right)$ as a function of frequency $\left.(\mathrm{f} / \mathrm{MHz}) ; \mathrm{C}\right)$ Numerical modelling of the 2-port box with an FDTD based 3D electromagnetic software, D) Simulated reflection and transmission parameters $\left(\left|\mathrm{S}_{\mathrm{ij}}\right| / \mathrm{dB}\right)$ as a function of frequency (f/MHz). 2-port $\mathrm{S}_{11}$ (blue solid line), 2-port $\mathrm{S}_{21}$ (red solid line), 3-port $\mathrm{S}_{11}$ (orange dashed line), 3-port $\mathrm{S}_{21}$ (purple dashed line)

\subsection{Frequency domain characterization}

\subsubsection{Experimental measurements}

The frequency capabilities of the 2 and 3-port coaxial boxes were assessed with electromagnetic measurements and numerical simulations. The scattering parameters measurements aim to characterize connectors (HN, THT) and boxes frequency behavior and limitations. For the frequency characterization, the PCSS was replaced by a short wire (Fig. 2A). Port \#3 is connected to the high voltage source though a $330 \mathrm{k} \Omega$ resistor (under the red rubber). Due to the high resistor soldered in contact with the inner conductor, port \#3 is almost equivalent to an open circuit for high frequencies and it allows isolating the high-voltage power supply from reflected pulses. Typical high frequency characteristics i.e., S-parameters of the boxes' ports 1 and 2 were measured from $1 \mathrm{MHz}$ to $3 \mathrm{GHz}$ with a vector network analyzer (VNA 8753E, HP, USA). From $S_{i j}$ parameters, i.e. the transmission coefficients $\left(S_{21}\right.$ and $\left.S_{12}\right)$, the ability to transmit wide band signals 
such as ultra-short duration pulses trough the boxes was assessed. Fig. $2 \mathrm{~B}$ presents the reflection $\mathrm{S}_{11}$ and transmission $\mathrm{S}_{21}$ coefficients of the 2-port and 3-port coaxial boxes. From the reflection coefficient $\mathrm{S}_{11}$, the bandwidth at $-20 \mathrm{~dB}$ which corresponds to a $10 \%$ reflected pulse voltage is $350 \mathrm{MHz}$. The reflection and transmission coefficients evidence overall low losses and good transmission of $10 \mathrm{~ns}$ duration pulses.

\subsubsection{Numerical modelling of the generator}

As illustrated in Fig. 2C, D, the 2-port coaxial box was numerically modelled using an in-house 3D electromagnetic software based on a Finite Difference Time Domain (FDTD) algorithm [31], [36]-[41]. All metallic parts of the box, connectors and electrodes were modeled as perfect electric conductors. The internal dielectric of the THT, HN connectors and the coaxial cables made of Teflon (polytetrafluoroethylene - PTFE) was modelled with a dielectric constant of 2.1. The box was filled with vegetable oil modelled with a dielectric constant of 2.5. The results of the simulated reflection coefficient show a $-20 \mathrm{~dB}$ bandwidth of $350 \mathrm{MHz}$, which is consistent with the experimental results obtained for the 2-port and 3-port boxes measurements. The frequency bandwidth is mainly limited by the THT connectors.

\subsection{In vitro experiments 2.3.1 Cell culture}

The human glioblastoma cell line U87-MG (ATCC HTB-14) was cultured in T25 flasks at $37^{\circ} \mathrm{C}, 5 \% \mathrm{CO}_{2}$ in DMEM + glutamax medium (Gibco) supplemented with 10\% FBS (Gibco), $100 \mathrm{U} / \mathrm{ml}$ penicillin and $100 \mu \mathrm{g} / \mathrm{ml}$ streptomycin (Gibco), 0.075\% sodium bicarbonate (Gibco) and 1\% MEM non-essential amino acids solution (Gibco). On reaching $80 \%$ confluence, cells were detached from the surface of the flask by washing twice with PBS (Gibco) and then incubating for $3-5$ minutes at $37^{\circ} \mathrm{C}$ with trypsin (Gibco). Trypsin activity was stopped with the addition of cell culture media and then centrifuged for 3 minutes at $600 \mathrm{~g}$. The resulting cell pellet was resuspended in the cell culture media for use in experiments and for reseeding flasks.

\subsubsection{Live cell imaging and nsPEF exposure}

Cells were plated at a density of $1.8 \times 10^{5}$ cells $/ \mathrm{ml}$ into $35 \mathrm{~mm}$ dishes, each containing a $22 \mathrm{~mm}$ poly-L-lysine (Sigma-Aldrich) coated glass coverslip, and incubated for 36-48 hours. Imaging coverslips were removed from their dishes, sandwiched into plastic imaging chambers and covered in $900 \mu \mathrm{l}$ of room temperature HEPES-buffered salt solution (HBSS) ( $\mathrm{NaCl} 121 \mathrm{mM}, \mathrm{KCl} 5.4 \mathrm{mM}, \mathrm{MgCl}_{2} 0.8 \mathrm{mM}, \mathrm{NaHCO}_{3} 6 \mathrm{mM}$, D-glucose $5.5 \mathrm{mM}$, HEPES $25 \mathrm{mM}$, with either $\mathrm{CaCl}_{2} 1.8 \mathrm{mM}\left(\mathrm{Ca}^{2+} \mathrm{HBSS}\right)$ or EGTA $4 \mathrm{mM}\left(\mathrm{Ca}^{2+}\right.$ free HBSS), $\left.\mathrm{pH} 7.3\right)$ containing $2 \mu \mathrm{M}$ of YP (Life Technologies).

Cells were observed by epifluorescence using a Leica DMI6000 microscope with a 63x objective. Fluorescent excitation was provided by a Spectra 7 light engine (Lumencor). Emitted light was filtered and captured on an electronmultiplying charge-coupled device camera (EMCCD Evolve 512, Roper) with 512 x 512 pixels (YP excitation: $488 \mathrm{~nm}$; emission: 473-575 nm). The system was controlled by, and images were captured with, Metafluor (version 7.8 Molecular Devices). Images were acquired during $180 \mathrm{~s}$ every 3 seconds for a total of 60 images using a $2 \mathrm{x}$ binning. Specifically, cellular fluorescence was recorded $30 \mathrm{~s}$ before the nsPEF exposure to obtain baseline, and $150 \mathrm{~s}$ after the pulse application.

Cells were exposed to $10 \mathrm{~ns}$ unipolar, bipolar, and paired nsPEF with different durations of the interphase interval. For paired pulses, two delays, including 50 and 110 ns were considered, while for bipolar nsPEF 30, 40, 50, 70, 110, and $150 \mathrm{~ns}$ delays were used. Cells were also exposed to either bipolar or paired nsPEF with no delay between the two polarities. Sham exposures were performed under identical experimental conditions, but with the generator switched off. The peak amplitude of the first and second phases of the generated pulses was equal to $5.7 \mathrm{kV}$ corresponding to an $E$-field strength of $11.5 \mathrm{MV} / \mathrm{m}$. Pulses were delivered using an electrode delivery system, which allowed real-time observations with the microscope objective, and comprised of two steel electrodes, separated by a gap of $330 \mu \mathrm{m}$ and with $50 \Omega$ impedance in parallel. The matching resistor was a high-voltage $50 \Omega$ terminator (Model 2051, Barth Electronics, Inc, NV, USA). The electrodes were positioned so that they touched the coverslip. A fresh coverslip was used for every control and every nsPEF treated experiment. Image stacks from live cell imaging experiments were analysed using Image Analyst MKII (Image Analyst Software, Novato, CA). Images were background subtracted, and then regions of interest (ROIs) were drawn around each cell in the field of view. Mean fluorescence intensity for each ROI was recorded. 


\subsubsection{Statistical analysis}

At least 15 cells from 5 different experiments were exposed or sham-exposed for each experimental condition. Statistical analyses were performed using Origin 2018 (OriginLab Corporation, Northampton, MA, USA) software. Datasets were first tested for normal distribution using Kolmogrov-Smirnov test. Unpaired two-tailed t-test was used for the statistical comparisons of the data. For all tests, a $p$ value $<0.05$ was considered statistically significant. Results are presented as mean $\pm \mathrm{SE}$ (Standard Error of the Mean).

\section{Results and Discussion \\ 3.1 High-voltage nanosecond pulses}

Fig. 3 illustrates the ability of the implemented generator to produce unipolar, bipolar, and paired pulses, with a duration at the Full Width at Half Maximum (FWHM) of 10 ns. A 3-ns laser was used with energy per optical pulse of $18 \mathrm{~mJ}$. The bias voltage was $12 \mathrm{kV}$, which corresponds to the voltage used for experimental biological investigations. The rise time, considered between $10 \%$ and $90 \%$ of the pulse maximum amplitude, was of approximately $2.9 \mathrm{~ns}$. To obtain monopolar, bipolar, or paired pulses with no delay between the phases, the output of the port- 2 was directly connected to a $50 \Omega$ terminator, short circuit, or open circuit, respectively.

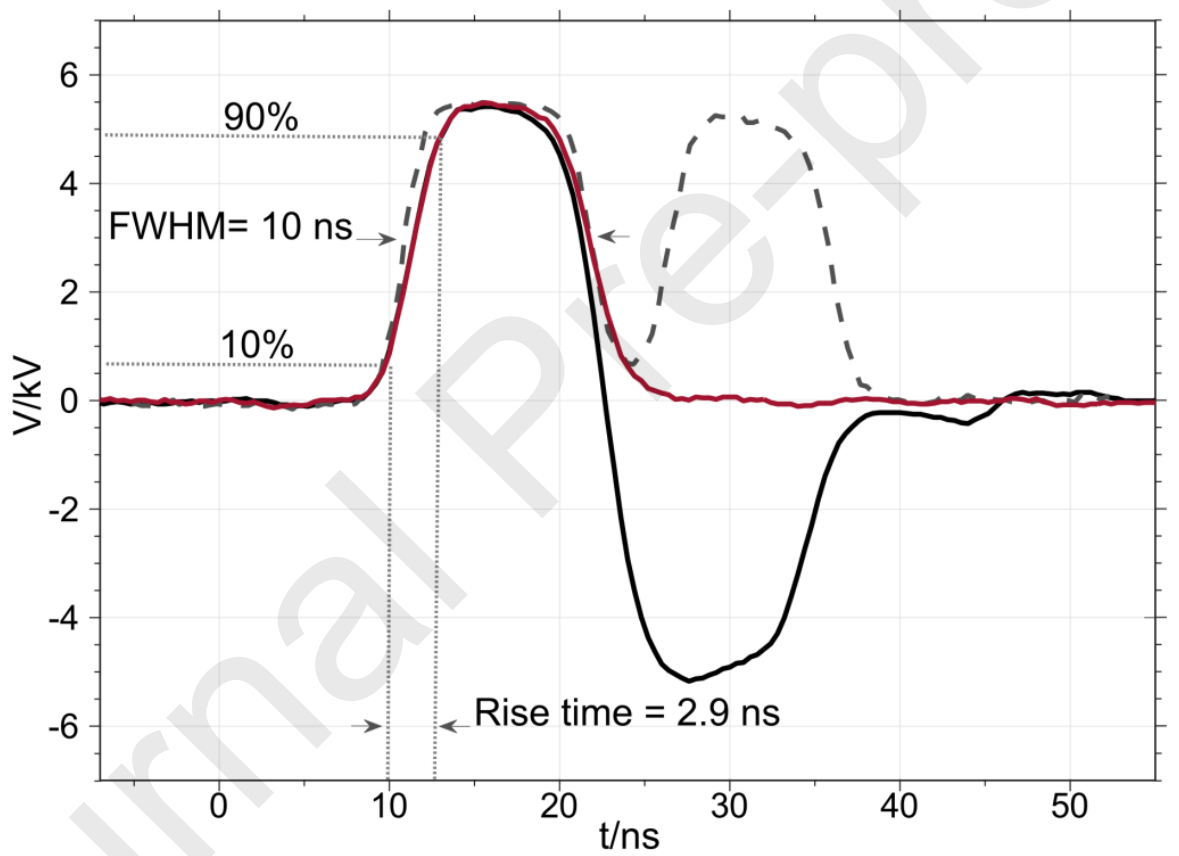

Fig. 3. Unipolar (red solid line), bipolar (black solid line), and paired (gray dashed line) voltage pulses. Voltage (V/kV) is presented as a function of time (t/ns). The bias voltage is set to $12 \mathrm{kV}$ and the optical energy is $18 \mathrm{~mJ}$ per pulse. The rise time is of $2.9 \mathrm{~ns}$ and the Full Width at Half Maximum (FWHM) of $10 \mathrm{~ns}$.

\subsection{High-voltage delayed nanosecond pulses}

The system developed allowed generating pulses with different delays and amplitudes, maintaining the rising time of both polarities of approximately $2.9 \mathrm{~ns}$ and duration at the FWHM of $10 \mathrm{~ns}$. By varying the length of the coaxial cable at the output of the 2-port coaxial box from approximately 0.5 to $35 \mathrm{~m}$, we have generated pulses with delays from 17 to $360 \mathrm{~ns}$. The delay is defined as the time interval between the onset of the first and the second pulses.

Fig. 4 shows bipolar and paired pulses obtained for eight cable lengths of $0.5,5,10,16,20,25,30$ and $35 \mathrm{~m}$ inserting a delay of $17,55,110,170,210,260,310,360 \mathrm{~ns}$, respectively. For both bipolar and paired pulses, the amplitude of the second phase decreased by $0.02 \mathrm{~dB} / \mathrm{m}$ when a delay was applied. The amplitude decrease is due to the residual resistor losses, less than $5 \%$ of the maximum theoretical voltage, existing when the second pulse is propagating through the PCSSs. These losses slightly increase over time due to propagation along the cable and due 
to cable losses. The losses increase with the cable length. Therefore, for biological investigations we only considered delays up to $150 \mathrm{~ns}$ where the second polarity decrease stays within an acceptable range ( $10 \%-15 \%$ of the first polarity).

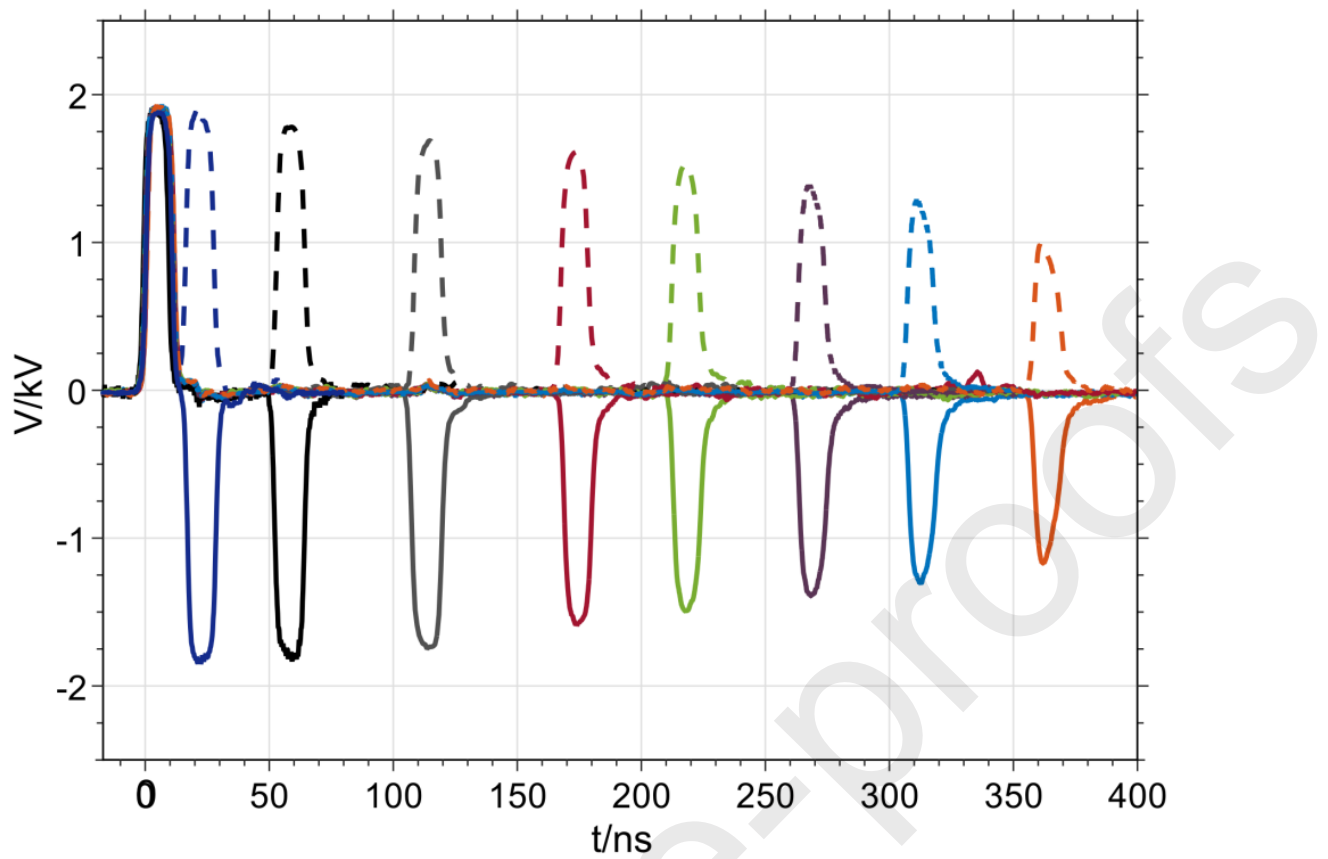

Fig. 4. Bipolar (solid lines) and paired (dashed lines) pulses with delay of $17 \mathrm{~ns}$ (dark blue), $55 \mathrm{~ns}$ (black), $110 \mathrm{~ns}$ (gray), $170 \mathrm{~ns}$ (red), $210 \mathrm{~ns}$ (green), $260 \mathrm{~ns}$ (purple), $310 \mathrm{~ns}$ (sky blue), and $360 \mathrm{~ns}$ (orange) for the bias voltage of $4 \mathrm{kV}$ and optical energy of $18 \mathrm{~mJ}$ per pulse. The rise time was of 2.9 ns and the Full Width at Half Maximum (FWHM) of $10 \mathrm{~ns}$. Voltage $(\mathrm{V} / \mathrm{kV})$ are presented as a function of time $(\mathrm{t} / \mathrm{ns})$

\subsection{Delivery system electromagnetic modelling and simulations}

Fig. 5A illustrates the modelling of the electrodes delivery system used to apply nsPEF to U87-MG cells. The electrodes, modeled as perfect electric conductors, are connected through a Tee adaptor to the generator and to a $50 \Omega$ high-voltage resistor placed in parallel to ensure impedance matching with the generator. The impedance of the electrodes delivery systems is higher than one hundred $\Omega$ [1], [8], [10], [40], [42], [43]. Thus, although the applied power is mainly dissipated in the $50 \Omega$ resistor, the electric field applied between the electrodes is more prevalent in terms of cell perturbation. The electrodes are $4 \mathrm{~mm}$ long, $2 \mathrm{~mm}$ wide and the interelectrode gap is $330 \mu \mathrm{m}$. The electrical properties of the biological pulsing medium, extracted from experimental measurements, were a dielectric constant of 72 and an electrical conductivity of $1.5 \mathrm{~S} / \mathrm{m}$, at low frequency and room temperature. For the 10-ns pulses generated in this study, the main part of the frequency spectrum is contained in a $100-\mathrm{MHz}$ bandwidth (Fig. 5D). Over this bandwidth the electrical properties of the biological pulsing medium can be considered constant.

In the simulations, all the dielectric parameters were considered constant over the frequency range of interest and a $30 \mu \mathrm{m}$ grid size was used to model the interelectrode gap. Fig. 5B shows the electric field distribution along a horizontal cut at the bottom of the pulsing suspension and along a vertical cut at the electrodes bending position. The electric field temporal distribution and frequency spectrum at the exposed cells position for both unipolar and bipolar pulses are shown in Fig. 5C and 5D. The transfer function of the electrodes delivery system was extracted from 3D EM simulations. The electric field was calculated using the measured pulse voltage generated (port \#1) as input of the transfer function. The electric field distribution plotted during the pulse plateau at $8 \mathrm{~ns}$ is homogeneous between the electrodes. The intensity at the exposed area was $11.5 \mathrm{MV} / \mathrm{m}$ for $5.7 \mathrm{kV}$ generated voltage. As observed from the electric field frequency spectrum, the main transmitted energy, i.e. for $-20 \mathrm{~dB}$ level, is within $100 \mathrm{MHz}$ bandwidth. The applied electric field spectrum is comprised in the $350 \mathrm{MHz}$ frequency bandwidth obtained from $\mathrm{S}_{11}$ parameters of the 2-port and 3-port coaxial boxes (Fig. 2B). This indicates that the boxes and their high-voltage connectors are well matched for delivering 10 -ns duration pulses. 

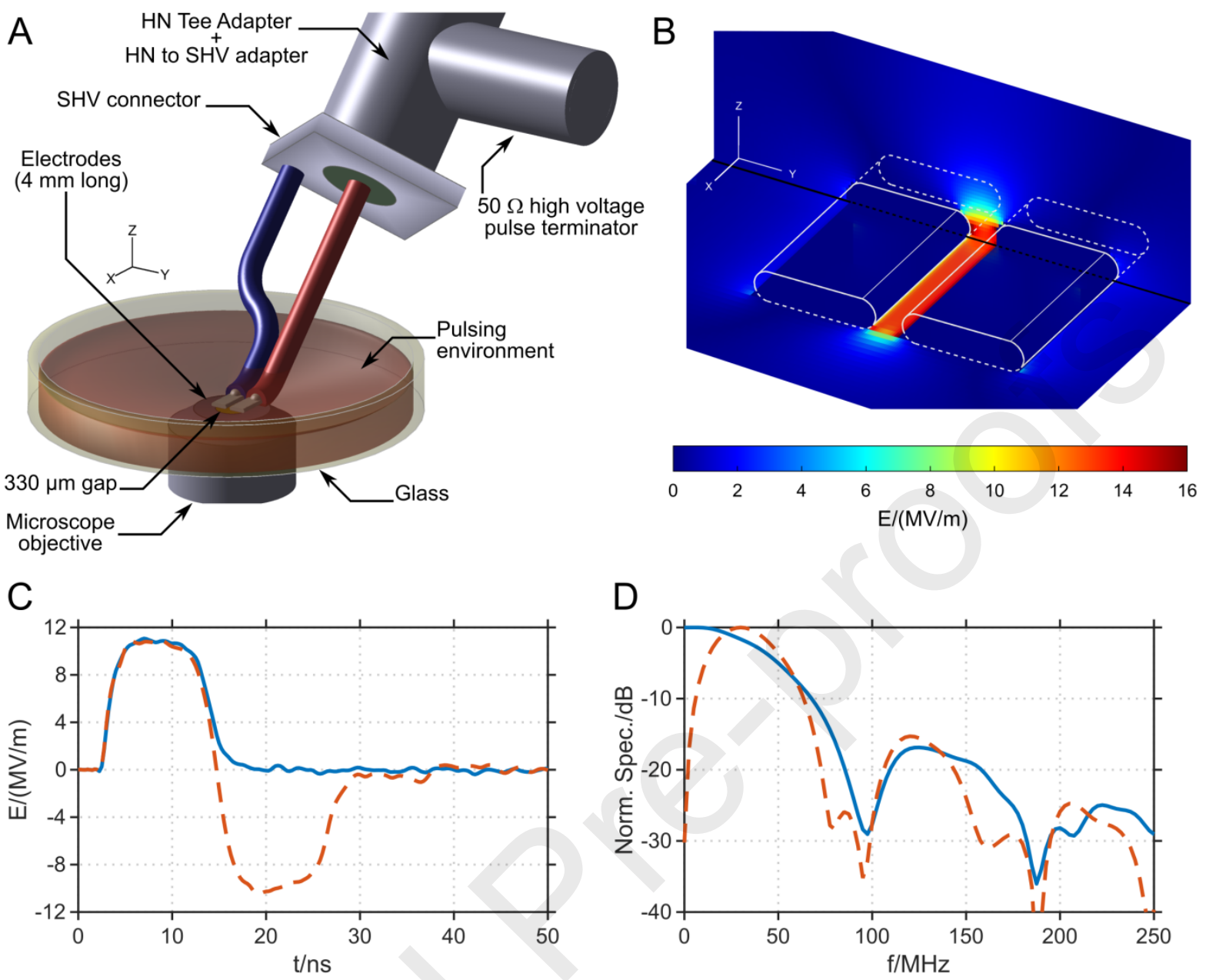

Fig. 5. Electrodes delivery system used to apply nsPEF to U87-MG cells for a $5.7 \mathrm{kV}$ output generated pulse voltage extracted from experimental measurements. A) Numerical modelling of the electrodes, pulsing environment, connectors, and adaptors to $50 \Omega$ pulse terminator and the generator. B) Electric field distribution (E/(MV/m)) extracted from 3D EM simulations along a horizontal cut at the bottom of the pulsing suspension (exposed cells position) and along a vertical cut close to the electrodes bending position. Electric field (unipolar and bipolar) extracted from 3D EM simulations in the middle of the interelectrode gap C) Electric field (E/(MV/m)) as a function of time (t/ns), and D) Normalized spectrum (Norm. $\mathrm{Spec} . \mathrm{dB}$ ) as a function of frequency (f/MHz). Unipolar nsPEF (blue solid line), bipolar nsPEF (red dashed line)

\subsection{Bipolar cancellation and enhancement of the YO-PRO ${ }^{\text {TM-1 }}$ uptake}

To verify the generator functionality and see the effect of these pulse forms on cell membranes, uptake of the dye YP into U87-MG cells was measured. YP is a dye that is excluded from cells with intact plasma membranes, and by measuring its uptake we were able to determine the extent of membrane poration [44]. Under all pulse conditions uptake of YP was observed immediately after exposure (the black arrows mark the delivery of the pulse), with dye uptake continuing throughout the rest of the imaging period (Fig. 6).

All paired pulse conditions resulted in significant higher uptake of YP than the unipolar pulse, with no significant difference due to the delay duration being found (Fig. 7). Plotting the curve integral rather than the final uptake at $180 \mathrm{~s}$ avoided random fluctuations in the last point and captures a cumulative effect. Bipolar pulses with no delay or a very short delay ( $30 \mathrm{~ns}$ ) showed significantly lower dye uptake than the unipolar pulse. All other bipolar conditions showed an enhanced uptake, linked to delay duration that, from $110 \mathrm{~ns}$ delay, attains the level of paired pulses. It can be noted that the uptake enhancement gets stronger for longer paired pulses delays. Or, conversely, the shorter the delay between 
paired pulses and the less effective they are. This suggests that there are two competing mechanisms, cancellation and enhancement, which for larger pulse intervals both result in higher uptake.

Consistently with previous studies [2], [9], the bipolar pulse with no delay showed less YP uptake than the unipolar pulse, i.e., bipolar cancellation. Reduction of this cancellation has been observed when a delay is introduced between the two phases of the pulse, however delays of $\mu$ s duration still result in less uptake than a unipolar pulse [1], [4], [8]. To our knowledge, the effect, on cells, of delays that are less than $100 \mathrm{~ns}$ has not previously been explored, nor has the effect of bipolar delay on pulses that have a duration of less than $300 \mathrm{~ns}$. We show that for $10 \mathrm{~ns}$ pulses bipolar cancellation only occurs for very short (30 ns) delays. Longer delays, of between 40 and $150 \mathrm{~ns}$, result in a significant enhanced uptake when compared to the unipolar pulse. This suggests that the second phase has an additive effect on the first, which increases with delay duration. By a delay of $110 \mathrm{~ns}$ this additive effect is equal to applying two positive pulses.
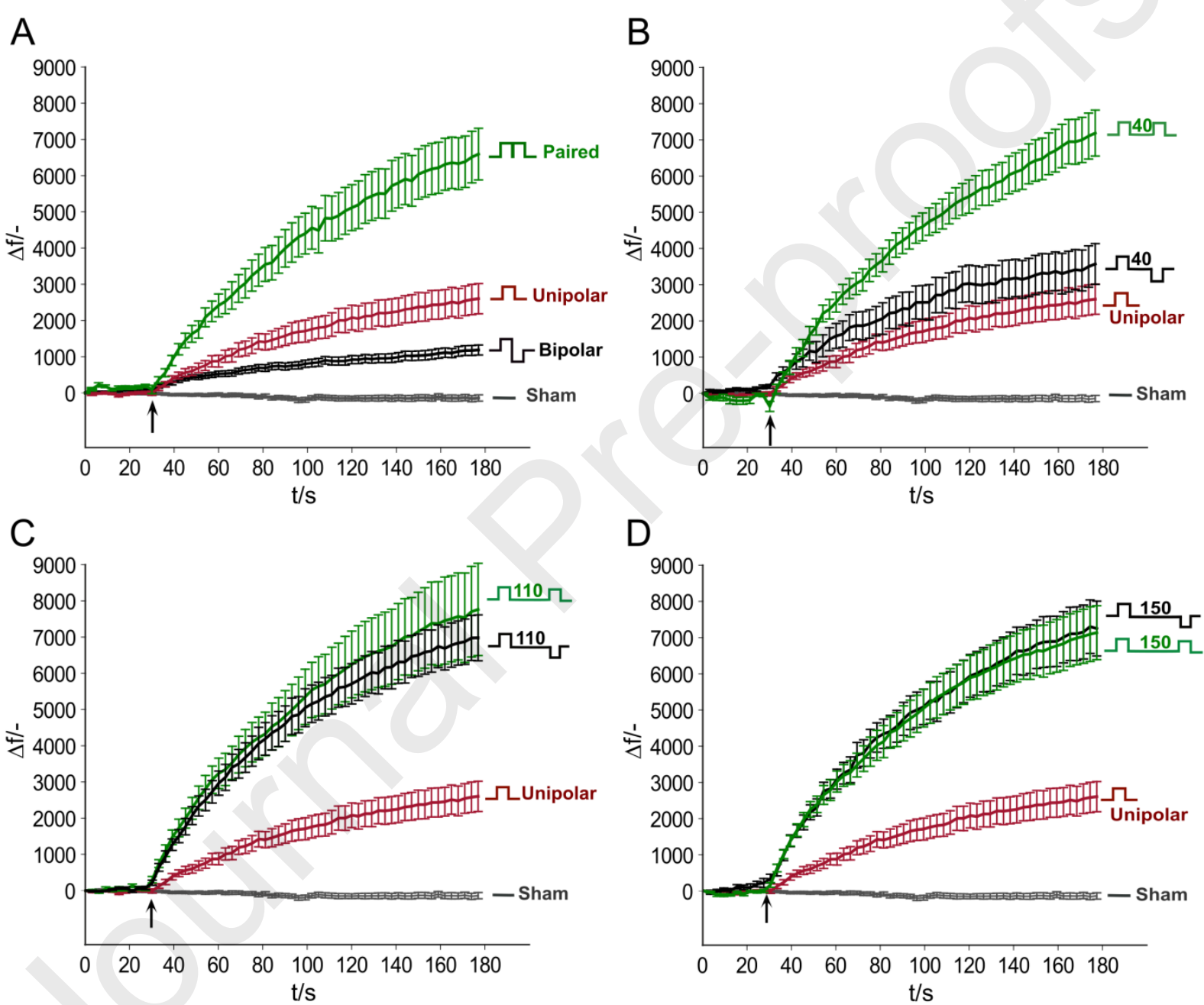

Fig. 6. Change in fluorescence $(\Delta \mathrm{f} /-)$ over time $(\mathrm{t} / \mathrm{s})$ showing YO-PRO ${ }^{\mathrm{TM}}-1$ uptake into U87-MG cells following the application of a single, $10 \mathrm{~ns}$ unipolar (red lines), bipolar (black lines) or paired pulse (green lines) with A) no delay, B) $50 \mathrm{~ns}, \mathrm{C}) 110 \mathrm{~ns}$, and D) $150 \mathrm{~ns}$ delays with an electric field strength of $11.5 \mathrm{MV} / \mathrm{m}$. Results for sham exposure are represented by the gray lines. The black arrows mark the start of the pulse.

An electrical model aiming to explain the mechanism of bipolar cancellation for pulses with typical durations between 0.1-1 $\mu$ s was proposed [1], [8] [10]. The model assumes that the presence of a back pulse, opposite in polarity compared to the front pulse, determines a switch of the electrical phenomena occurring at the location of the cellular membrane. However, the delivery of the back-pulse causes attenuation of the charging effects driven by the front pulse. This results in the decrease of the time interval within the membrane potential overcomes the YP uptake threshold, and therefore in a lower YP uptake compared to the one induced by the unipolar pulse [10]. Increasing the delay between the front and the back pulse, modifies the charging effects due to the electrodes switch. Indeed, more YP 
uptake is observed on the "cathode" side of the cell compared to the approximately equivalent distribution between "anode" and "cathode" sides of bipolar pulses without delay [8].

For shorter pulses durations, without charging effect, our results are also consistent with this behavior. The charging phenomena is becoming less efficient in terms of electroporation. For short-duration and short-interval pulses, a cell is more permeable to the electric field. Indeed, when the second polarity pulse is delivered within a delay sufficiently short, i.e., $30 \mathrm{ns,} \mathrm{we} \mathrm{observed} \mathrm{cancellation} \mathrm{(i.e.,} \mathrm{YP} \mathrm{uptake} \mathrm{is} \mathrm{nearly} \mathrm{the} \mathrm{half} \mathrm{compared} \mathrm{to} \mathrm{the} \mathrm{unipolar}$ response). However, when we enlarged the interphase interval to values $>40 \mathrm{~ns}$, the cancellation effect disappears. The threshold for bipolar cancelation "cancelation" is approximately $40 \mathrm{~ns}$ i.e. 4 times the duration of the pulses. Differences in terms of YP uptake become statistically significant compared to the unipolar pulse for duration of the interphase interval $\geq 70 \mathrm{~ns}$. Numerical simulations, accounting for the amount of a tracer entering the cells [45], seem also to support our results. When the interphase interval is short, external molecules assisted by the back pulse, enter the pores created by the front pulse, occluding the passage of YP, thereby cancelling the effect previously elicited by the first polarity of the pulse. The effect is progressively reversed for longer duration of the interphase interval due to a delay in the occlusion phenomenon.

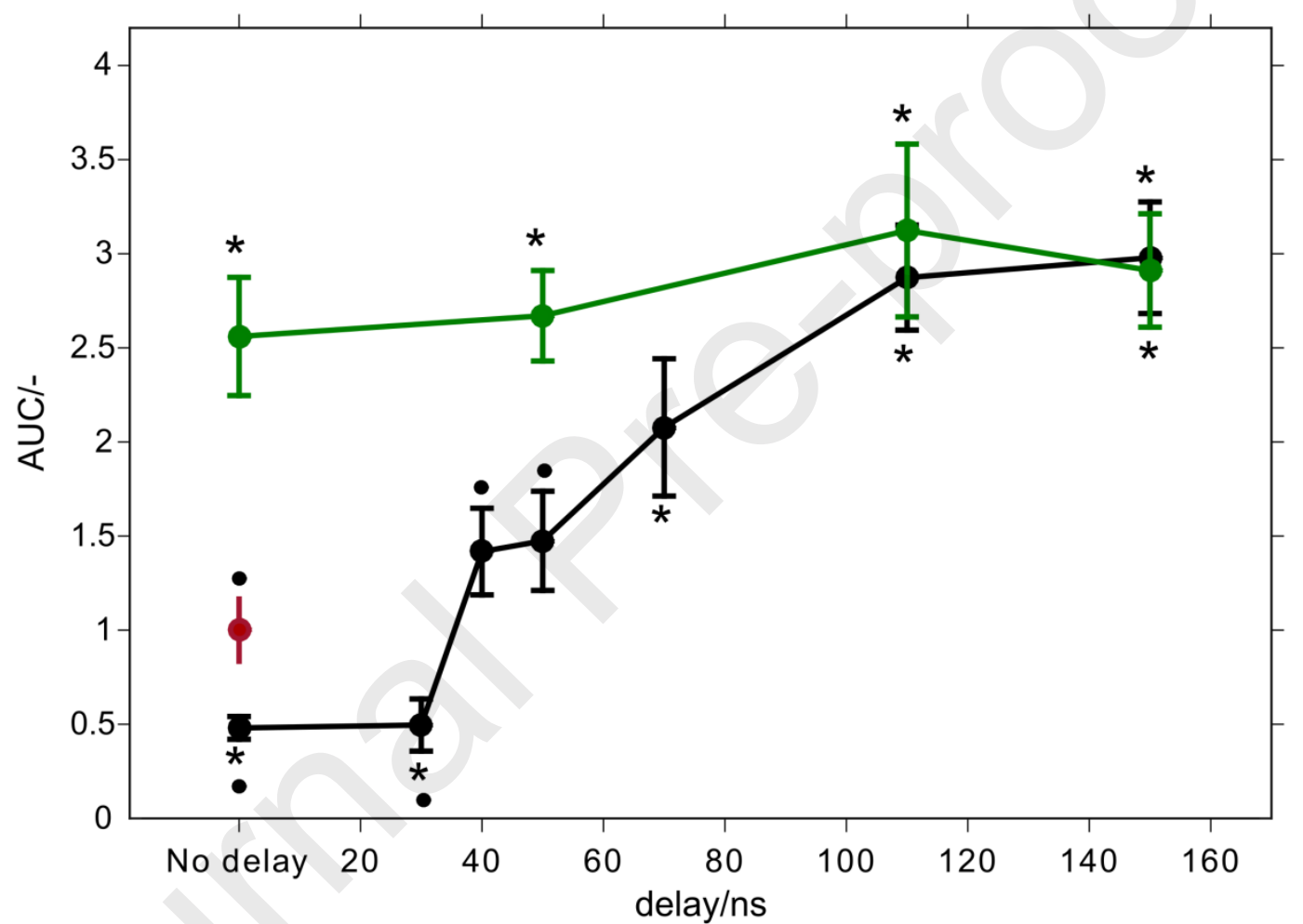

Fig. 7. The area under the fluorescence curves (AUC/-) of Fig. 6 plotted as a function of the delay (delay/ns) between pulses (no delay and 30, 40, $50,70,110$ and $150 \mathrm{~ns}$ ), normalized to the unipolar value: unipolar (red dot), bipolar (black dot) and paired (green dot) pulses. The AUC plots show the cumulative effect of dye uptake and are influenced by maximum uptake as well as uptake dynamics. For all conditions average \pm SE (Standard Error of the Mean) is shown from a minimum of 15 cells from 5 different experiments. * indicates statistical significance at $p<0.05$ versus unipolar nsPEF. $\bullet$ indicates statistical significance at $\mathrm{p}<0.05$ versus paired (all delays) nsPEF.

Note that, a delay of $100 \mathrm{~ns}$ has previously been shown, when using a single $300 \mathrm{~ns}$ bipolar pulse, to cause the same amount of cancellation as a bipolar pulse with no delay [4]. The reason behind this difference between 10 and $300 \mathrm{~ns}$ pulses remains to be determined but may be linked to the relationship between pulse duration, field strength, and the charging time constant of the cell. In principle, membrane charging/discharging time and bipolar cancellation efficiency could be modulated by changing the suspension medium conductivity. However, although bipolar cancellation was found to be stronger in low conductivity media, no significant differences in its time dependence were observed with respect to high conductivity media for 300 ns pulses [4].

\section{Conclusion}

In this work, a robust high-voltage generator able to produce unipolar, bipolar, and paired $10 \mathrm{~ns}$ pulses with different delays among the polarities was developed and fully characterized. The proposed generator is based on the 
frozen-wave principle, where the energy of a power generator stored into a transmission line is delivered after a $2.4 \mathrm{~ns}$ pulsed laser optically triggers two PCSSs. The proposed generator is highly versatile allowing the production of pulses with different amplitudes, durations, interphase intervals, and shape by varying the bias voltage (4-12 kV), the coaxial cable length $(0-35 \mathrm{~m})$, and its final connection $50 \Omega$ terminator, open or short circuit). The generator's main originality consists in obtaining a very short delay between the pulse polarities in the ns range, allowing investigation of cellular responses beyond the time intervals considered so far (i.e., $100 \mathrm{ns)} \mathrm{[4].} \mathrm{The} \mathrm{generator} \mathrm{was} \mathrm{characterized} \mathrm{experimentally}$ and numerically in the frequency and time domains. The generator frequency bandwidth was assessed to $350 \mathrm{MHz}$ at $-20 \mathrm{~dB}$ threshold of the reflection coefficient. The electric field temporal distribution at the level of the exposed cells demonstrates the ability of the delivery system to apply $10 \mathrm{~ns}, 11.5 \mathrm{MV} / \mathrm{m}$ nsPEF with a $5.7 \mathrm{kV}$ output generated voltage for biological investigations.

In vitro biological experiments were performed on the U87-MG glioblastoma cell line. Pulses were delivered to the cells through a couple of electrodes with $330 \mu \mathrm{m}$ gap. Membrane poration was evaluated by means of YP uptake following the exposure to $10 \mathrm{~ns}$ unipolar, bipolar, and paired pulses with different delays between the polarities (30$150 \mathrm{~ns}$ ). Our results showed that cancellation of the YP uptake was observed only after bipolar pulses with no or very short delay of $30 \mathrm{~ns}$, and the phenomenon was completely reversed when the delay was increased between 70 and $150 \mathrm{~ns}$ (significant differences compared to unipolar). In this interval, the YP uptake was between 2 and 3 times stronger compared to the effect induced by unipolar nsPEF, as opposed to literature data where cancellation was observed for pulse durations as long as $50 \mu$ s [4]. However, additive effects were observed following the exposure to paired pulses, in agreement with previous studies [4]. In this case, YP uptake was up to 3 times stronger than the one observed following the unipolar response and very similar to the response obtained for duration of the bipolar pulses in the $70-150$ ns range.

Further perspectives of the current study involve the possibility to evaluate larger pulse durations, between 10 and $60 \mathrm{~ns}$, as well as the quantification of the transmembrane induced potential, with the aim to provide new insights for the understanding of the complex phenomenon of bipolar cancellation.

\section{Authors contributions:}

DAC and PL designed, conceived the generator and the study. RO and LC contributed equally to the acquisition, analysis, and interpretation of data. DAC and PL contributed to the acquisition and interpretation of data and numerical modeling. CP contributed to revising the paper critically for important intellectual content. All authors contributed to drafting the manuscript. All authors read and gave approval of the final version to be submitted.

\section{Acknowledgements}

This project has received funding from the European Union's Horizon 2020 research and innovation program under grant agreement No 737164. 


\section{References}

[1] A. G. Pakhomov et al., Cancellation of cellular responses to nanoelectroporation by reversing the stimulus polarity, Cell. Mol. Life Sci. 71(2014) 4431-4441.

[2] E. C. Gianulis, J. Lee, C. Jiang, S. Xiao, B. L. Ibey, A. G. Pakhomov, Electroporation of mammalian cells by nanosecond electric field oscillations and its inhibition by the electric field reversal, Sci Rep 5(2015) 5:13818.

[3] B. L. Ibey et al., Bipolar nanosecond electric pulses are less efficient at electropermeabilization and killing cells than monopolar pulses, Biochemical and Biophysical Research Communications 443(2014) 568-573.

[4] E. C. Gianulis, M. Casciola, S. Xiao, O. N. Pakhomova, A. G. Pakhomov, Electropermeabilization by uni- or bipolar nanosecond electric pulses: The impact of extracellular conductivity, Bioelectrochemistry 119(2018) 1019.

[5] T. R. Bagalkot, N. Leblanc, G. L. Craviso, Stimulation or cancellation of Ca2+ influx by bipolar nanosecond pulsed electric fields in adrenal chromaffin cells can be achieved by tuning pulse waveform, Sci Rep 9 (2019) 11545 .

[6] M. Casciola et al., Cancellation of nerve excitation by the reversal of nanosecond stimulus polarity and its relevance to the gating time of sodium channels, Cell. Mol. Life Sci. 76 (2019) 4539-4550.

[7] K. H. Schoenbach, A. G. Pakhomov, I. Semenov, S. Xiao, O. N. Pakhomova, B. L. Ibey, Ion transport into cells exposed to monopolar and bipolar nanosecond pulses, Bioelectrochemistry 103(2015) 44-51.

[8] C. M. Valdez, R. Barnes, C. C. Roth, E. Moen, B. Ibey, The interphase interval within a bipolar nanosecond electric pulse modulates bipolar cancellation, Bioelectromagnetics 39(2018) 441-450.

[9] E. B. Sözer, P. T. Vernier, Modulation of biological responses to $2 \mathrm{~ns}$ electrical stimuli by field reversal, Biochimica et Biophysica Acta (BBA) - Biomembranes 1861(2019) 1228-1239.

[10] C. M. Valdez, R. A. Barnes, C. C. Roth, E. K. Moen, G. A. Throckmorton, B. L. Ibey, Asymmetrical bipolar nanosecond electric pulse widths modify bipolar cancellation, Sci Rep 7(2017) 16372.

11] A. G. Pakhomov, S. Grigoryev, I. Semenov, M. Casciola, C. Jiang, S. Xiao, The second phase of bipolar, nanosecond-range electric pulses determines the electroporation efficiency, Bioelectrochemistry(2018) 123-133.

[12] I. Semenov, M. Casciola, B. L. Ibey, S. Xiao, A. G. Pakhomov, Electropermeabilization of cells by closely spaced paired nanosecond-range pulses, Bioelectrochemistry 121(2018) 135-141.

[13] M. B. Sano et al., Bursts of bipolar microsecond pulses inhibit tumor growth, Sci Rep 5(2015) 14999.

[14] T. Kotnik, L. M. Mir, K. Flisar, M. Puc, D. Miklavčič, Cell membrane electropermeabilization by symmetrical bipolar rectangular pulses: Part I. Increased efficiency of permeabilization, Bioelectrochemistry 54(2001) 83-90.

[15] T. Kotnik, D. Miklavcic, L. M. Mir, Cell membrane electropermeabilization by symmetrical bipolar rectangular pulses. Part II. Reduced electrolytic contamination, Bioelectrochemistry 54(2001) 91-95.

[16] C. B. Arena, M. B. Sano, M. N. Rylander, R. V. Davalos, Theoretical considerations of tissue electroporation with high-frequency bipolar pulses," IEEE Transactions on Biomedical Engineering 58(2011) 1474-1482.

[17] S. Xiao, C. Zhou, E. Yang, S. R. Rajulapati, Nanosecond bipolar pulse generators for bioelectrics, Bioelectrochemistry 123(2018) 77-87.

[18] S. Kohler, V. Couderc, R. P. O'Connor, D. Arnaud-Cormos, P. Leveque, A versatile high voltage nano- and subnanosecond pulse generator, IEEE Transactions on Dielectrics and Electrical Insulation 20(2013) 1201-1208.

[19] M. Sack, M. Hochberg, G. Mueller, Design of a semiconductor-based bipolar Marx generator, IEEE International Power Modulator and High Voltage Conference (IPMHVC) (2014) 664-667.

[20] M. A. Elgenedy, A. M. Massoud, S. Ahmed, B. W. Williams, J. R. McDonald, A modular multilevel voltageboosting Marx pulse-waveform generator for electroporation applications, IEEE Transactions on Power Electronics 34(2019) 10575-10589.

[21] T. Heeren, J. T. Camp, J. F. Kolb, K. H. Schoenbach, S. Katsuki, H. Akiyama, 250 kV sub-nanosecond pulse generator with adjustable pulse-width, IEEE Transactions on Dielectrics and Electrical Insulation 14(2007) 884888.

[22] D. Zhang, Y. Zhou, J. Wang, P. Yan, A compact, high repetition-rate, nanosecond pulse generator based on magnetic pulse compression system, IEEE Transactions on Dielectrics and Electrical Insulation 18(2011) 11511157.

[23] T. Tang, F. Wang, A. Kuthi, M. A. Gundersen, Diode opening switch based nanosecond high voltage pulse generators for biological and medical applications, IEEE Transactions on Dielectrics and Electrical Insulation 14(2007) 878-883.

[24] M. Rebersek et al., Blumlein configuration for high-repetition-rate pulse generation of variable duration and polarity using synchronized switch control, IEEE Transactions on Biomedical Engineering 56(2009) 2642-2648.

[25] S. Romeo, M. Sarti, M. R. Scarfi, L. Zeni, Modified Blumlein pulse-forming networks for bioelectrical applications, J. Membr. Biol. 236(2010), 55-60. 
[26] J. F. Kolb et al., Nanosecond pulse generator with variable pulse duration for the study of pulse induced biological effects, IEEE International Power Modulators and High-Voltage Conference (2008) 61-64

[27] I.W. Davies et al., Push- pull configuration of high-power MOSFETs for generation of nanosecond pulses for electropermeabilization of cells, Int. J. Microw. Wirel. T. 11(2019) 645-657.

[28] E. Pirc, D. Miklavcic, M. Rebersek, Nanosecond pulse electroporator with silicon carbide mosfets: development and evaluation, IEEE Trans. Biomed. Eng. 66(2019) 3526-3533.

[29] D. Arnaud-Cormos, V. Couderc, P. Leveque, Photoconductive switching for pulsed high-voltage generators, in Handbook of Electroporation, D. Miklavcic, Ed. Cham: Springer International Publishing (2017) 1-21.

[30] M. Rebersek, D. Miklavcic, C. Bertacchini, M. Sack, Cell membrane electroporation-Part 3: the equipment, IEEE Electr. Insul. Mag 30(2014) 8-18.

[31] L. Carr, S.M. Bardet, R.C. Burke, D. Arnaud-Cormos, P. Leveque, R.P. O'Connor, Calcium-independent disruption of microtubule dynamics by nanosecond pulsed electric fields in U87 human glioblastoma cells, Sci Rep 7 (2017) 41267.

[32] L. Carr, S. M. Bardet, D. Arnaud-Cormos, P. Leveque, R. P. O'Connor, Visualization of an nsPEF induced calcium wave using the genetically encoded calcium indicator GCaMP in U87 human glioblastoma cells, Bioelectrochemistry 119(2018) 68-75.

[33] J. Dermol-Černe et al., Plasma membrane depolarization and permeabilization due to electric pulses in cell lines of different excitability, Bioelectrochemistry 122(2018), 103-114.

[34] S. El Amari, A. De Angelis, D. Arnaud-Cormos, V. Couderc, P. Leveque, Characterization of a linear photoconductive switch used in nanosecond pulsed electric field generator, IEEE Photonics Technology Letters 23(2011) 673-675.

[35] S. Kohler, R. P. O'Connor, T. D. T. Vu, P. Leveque, D. Arnaud-Cormos, Experimental microdosimetry techniques for biological cells exposed to nanosecond pulsed electric fields using microfluorimetry, IEEE T. Microw. Theory 61(2013) 2015-2022.

[36] A. Taflove, S. C. Hagness, Computational electrodynamics the finite-difference time-domain method, Boston Artech House (2005).

[37] P. Leveque, A. Reineix, B. Jecko, Modelling of dielectric losses in microstrip patch antennas: application of FDTD method, Electronics Letters 28(1992) 539-541.

[38] P. Leveque, C. Dale, B. Veyret, J. Wiart, Dosimetric analysis of a 900-MHz rat head exposure system, IEEE T. Microw. Theory 52(2004) 2076-2083.

[39] D. E. Chafai et al., Microtubulecytoskeleton remodeling by nanosecond pulsed electric fields, Advanced Biosystems Early access.

[40] Y.-H. Wu, D. Arnaud-Cormos, M. Casciola, J. M. Sanders, P. Leveque, P. T. Vernier, Moveable wire electrode microchamber for nanosecond pulsed electric-field delivery, IEEE Transactions on Biomedical Engineering 60(2013) 489-496.

[41] A. Nefzi, L. Carr, C. Dalmay, A. Pothier, P. Leveque, D. Arnaud-Cormos, Microdosimetry using rhodamine B within macro- and microsystems for radiofrequency signals exposures of biological samples, IEEE T. Microw. Theory 68(2020) 1142-1150.

[42] D. Arnaud-Cormos, P. Leveque, Y.-H. Wu, J. M. Sanders, M. A. Gundersen, P. T. Vernier, Microchamber setup characterization for nanosecond pulsed electric field exposure, IEEE Trans Biomed Eng 58(2011) 1656-1662.

[43] A. G. Pakhomov, J. F. Kolb, J. A. White, R. P. Joshi, S. Xiao, K. H. Schoenbach, Long-lasting plasma membrane permeabilization in mammalian cells by nanosecond pulsed electric field (nsPEF), Bioelectromagnetics 28(2007) 655-663.

[44] T. B. Napotnik, Fluorescent indicators of membrane permeabilization due to electroporation, in Handbook of Electroporation, D. Miklavčič, Ed. Cham: Springer International Publishing (2017) 1305-1323.

[45] T. R. Gowrishankar, J. V. Stern, K. C. Smith, J. C. Weaver, Nanopore occlusion: A biophysical mechanism for bipolar cancellation in cell membranes, Biochemical and Biophysical Research Communications 503(2018) 1194-1199. 